\title{
BMJ Open Compliant flooring to prevent fall-related injuries: a scoping review protocol
}

\author{
Chantelle C Lachance, ${ }^{1,2}$ Michal P Jurkowski, ${ }^{1}$ Ania C Dymarz, ${ }^{3}$ Dawn C Mackey ${ }^{1,2}$
}

To cite: Lachance CC, Jurkowski MP, Dymarz AC, et al. Compliant flooring to prevent fall-related injuries: a scoping review protocol. BMJ Open 2016;6: 011757. doi:10.1136/bmjopen-2016011757

- Prepublication history and additional material is available. To view please visit the journal (http://dx.doi.org/ 10.1136/bmjopen-2016011757).

Received 2 March 2016 Revised 22 June 2016 Accepted 23 June 2016

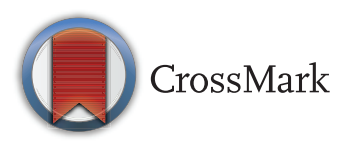

${ }^{1}$ Department of Biomedical Physiology and Kinesiology, Simon Fraser University, Burnaby, British Columbia, Canada

${ }^{2}$ Centre for Hip Health and Mobility, University of British Columbia, Vancouver, British Columbia, Canada

${ }^{3}$ W.A.C. Bennett Library, Simon Fraser University, Burnaby, British Columbia, Canada

Correspondence to Dr Dawn C Mackey; dmackey@sfu.ca

\section{ABSTRACT}

Introduction: Fall-related injuries can have serious consequences for older adults, including increased risk of dependence in daily activities and mortality. Compliant flooring is a passive intervention that may reduce the incidence and severity of fall-related injuries in healthcare settings, including acute and long-term care, but few sites have implemented compliant flooring, in part because synthesised evidence about key performance aspects has not been available.

Methods and analysis: We will conduct a scoping review to address the question: what is presented about the biomechanical efficacy, clinical effectiveness, cost-effectiveness, and workplace safety associated with compliant flooring systems that aim to prevent fall-related injuries? We will conduct a comprehensive and systematic literature search of academic databases (AgeLine, CINAHL, EBM Reviews, MEDLINE (Ovid), SportDiscus and Web of Science) and grey literature (clinical trial registries, theses/dissertations, abstracts/ conference proceedings and relevant websites). 2 team members will independently screen records (first titles and abstracts, then full text) and extract data from included records. Numerical and narrative analyses will be presented by theme (biomechanical efficacy, clinical effectiveness, cost-effectiveness, workplace safety).

Ethics and dissemination: This scoping review responds to the information needs of healthcare decision-makers tasked with preventing fall-related injuries. This review will summarise evidence about compliant flooring as a potential intervention for preventing fall-related injuries in older adults and identify gaps in evidence and new avenues for research. Results will be especially useful in long-term care, but also applicable in acute care, assisted living and home care. We will disseminate the review's findings via open-access publications, conference presentations, a webinar, a Stakeholder Symposium and a Knowledge-to-Action Report.

\section{INTRODUCTION}

Falls are the leading cause of injury-related hospitalisation and death among seniors and are responsible for annual direct costs of $\$ 3.4$ billion in Canada and $\$ 34$ billion in the USA. $^{1-3}$ Aside from death, hip fracture and traumatic brain injury (TBI) are the most

\section{Strengths and limitations of this study}

- This will be the first review to synthesise the available evidence about the biomechanical efficacy, clinical effectiveness, cost-effectiveness, and workplace safety associated with compliant flooring systems that aim to prevent fall-related injuries. Results will be relevant to long-term care, acute care, assisted living and home care.

- This scoping review will employ the widely used and accepted Arksey and 0'Malley ${ }^{28}$ framework and published updates to it, and it will include a comprehensive search of both academic and grey literature using a systematic approach.

- A limitation is that the grey literature search will be limited to the English language only.

- Consistent with the scoping review framework, the review will provide breadth of understanding about compliant flooring systems for fall-related injury prevention, but not depth of this topic, and nor will the quality of the included records be assessed.

serious fall-related injuries and are associated with lasting and devastating consequences, including loss of independence, reduced quality of life and premature mortality. ${ }^{4}$

The long-term care (LTC) setting is an especially high-risk environment for falls and fall-related injuries. Approximately $60 \%$ of LTC residents fall at least once per year, and $30 \%$ of falls in LTC cause injury, rates that are two to three times higher than for community-dwelling older adults. ${ }^{5-9}$ In fact, LTC residents are 10 times more likely to sustain a hip fracture than communitydwelling older adults, ${ }^{710}$ and following hospitalisation for hip fracture, $25 \%$ of LTC residents die within 6 months and $<50 \%$ of LTC residents regain their preinjury ambulation status. ${ }^{8} 11{ }^{12}$ Furthermore, TBIs have tripled in incidence over the past decade and account for almost half of the fatal falls in LTC residents. ${ }^{8} 13$ One-quarter of fall-related TBIs in older adults occur in LTC sites. ${ }^{13-15}$ 
The majority of fall-related TBIs are believed to occur as a result of the head directly striking another surface; thus, the severity of fall-related TBIs is influenced by the mechanical properties of the impact surface. ${ }^{16-18}$

Preventing falls and fall-related injuries among older adults in LTC remains a significant challenge, in part because LTC residents often present with compromised physical and cognitive function. ${ }^{19-21}$ A recent Cochrane systematic review showed that vitamin D supplementation leads to small reductions in fall rates in LTC, but concluded that there is insufficient evidence to comment on the potential effectiveness of other single or multifactorial fall prevention strategies in the LTC setting, including environmental, exercise, pharmaceutical and administrative interventions. ${ }^{19}$ When considering injury prevention strategies, evidence suggests that hip protectors reduce the risk of hip fracture by close to $80 \%$ when they are worn at the time of a fall; however, hip protectors only protect one impact site and poor adherence is a major barrier to effectiveness. ${ }^{22-25}$ Given the public health and individual burden caused by fallrelated injuries in LTC, it is essential to develop new and effective strategies for preventing fall-related injuries.

A promising strategy is to decrease the stiffness of the ground surface, and the subsequent forces applied to the body at impact, by installing compliant flooring, a passive intervention approach that (once installed) does not rely on user adherence. ${ }^{16}$ Unlike hip protectors, compliant flooring has the potential to reduce the frequency of all fall-related injuries, including hip and wrist fractures and TBIs. However, few LTC sites have implemented flooring systems specifically designed to reduce the severity of fall impacts. Healthcare decision-makers whom we have surveyed state that one barrier to uptake is a lack of synthesised evidence about key performance aspects of compliant flooring systems.

To inform fall-related injury prevention strategies in LTC, our goal is to conduct a scoping review to address the following research question: what is presented in the scientific literature about the biomechanical efficacy, clinical effectiveness, cost-effectiveness, and workplace safety associated with compliant flooring systems that aim to prevent fall-related injuries (table 1)? Our specific objectives are (1) to describe the extent, range and nature of research activity; (2) to identify gaps in research; and (3) to disseminate findings to stakeholders through effective knowledge translation.

\section{METHODS}

The scoping review methodology is particularly well matched to the research question because the use of compliant flooring for fall-related injury prevention is an emerging research area, the study objectives are defined broadly and exploratory in nature, and relevant evidence originates from published and unpublished sources from a variety of disciplines (eg, biomedical engineering, epidemiology, health economics, ergonomics) and study designs both in published and grey literature. $^{26}{ }^{27}$ Accordingly, we will follow the six-stage scoping review framework of Arksey and O'Malley ${ }^{28}$ and incorporate published amendments to it. ${ }^{26272930}$

\section{Identifying the research question}

Our research question was designed to generate breadth of coverage, ${ }^{28}$ and was developed originally by core members of the research team (CCL and DCM). It was then reviewed at a planning meeting with other members of the research team and by the study's Research Advisory Panel (composed of knowledge users) to further refine the types of information about compliant flooring that were of highest importance to knowledge users and researchers. The Panel input also helped develop the definition of compliant flooring systems and the four thematic areas of interest: biomechanical efficacy, clinical effectiveness, costeffectiveness and workplace safety (table 1).

\begin{tabular}{|c|c|}
\hline Concept & Definition \\
\hline $\begin{array}{l}\text { Compliant flooring } \\
\text { systems }\end{array}$ & $\begin{array}{l}\text { Broadly defined as flooring systems or floor coverings with some level of shock absorbency, for } \\
\text { example, safety flooring, shock absorbing flooring, dual stiffness flooring, rubber flooring, acoustic } \\
\text { flooring and carpet }\end{array}$ \\
\hline Biomechanical efficacy & $\begin{array}{l}\text { Evidence from laboratory research about (1) impact force attenuation or energy absorption during } \\
\text { real or simulated falls onto compliant flooring systems, or (2) gait performance, mobility } \\
\text { performance, assistive device use and/or balance on compliant flooring systems }\end{array}$ \\
\hline Clinical effectiveness & $\begin{array}{l}\text { Evidence from research involving human participants and measurement of how compliant flooring } \\
\text { systems affect falls and fall-related injuries }\end{array}$ \\
\hline Cost-effectiveness & $\begin{array}{l}\text { Evidence related to the costs of compliant flooring systems relative to their effects on fall and } \\
\text { fall-related injury healthcare costs }\end{array}$ \\
\hline Workplace safety & $\begin{array}{l}\text { Evidence about the effects of compliant flooring systems on musculoskeletal health and fatigue of } \\
\text { healthcare workers as a direct result of differences in floor compliance }\end{array}$ \\
\hline Fall-related injury & $\begin{array}{l}\text { Broadly defined as fractures or soft tissue injuries (eg, haematoma, traumatic brain injury, } \\
\text { dislocation, laceration/cut, sprain/strain, contusion/bruise, swelling, pain) as a direct result of impact } \\
\text { from a fall }\end{array}$ \\
\hline
\end{tabular}


Identifying relevant records

Licensed academic databases

Systematic searches will be conducted in the following licensed electronic databases: AgeLine, CINAHL Complete, EBM Reviews (includes CENTRAL, Cochrane Database of Systematic Reviews and DARE), Ergo-Abs (Ergonomics Abstracts), MEDLINE (Ovid), SPORTDiscus and Web of Science (table 2). The main concepts of our research question (ie, flooring, biomechanical efficacy, clinical effectiveness, costeffectiveness and workplace safety) will be expressed through a combination of keyword synonyms, related terms and controlled vocabulary terms (eg, MeSH). Our initial search strategy was developed using MEDLINE (Ovid; see online supplementary file 1) and will be refined in the light of the iterative nature of the search process. The final MEDLINE (Ovid) search string will guide the search process for other databases and inform

Table 2 Sources of academic and grey literature

\section{Search type \\ (date range searched)}

Academic search

(inception to present)

Grey literature search

(1990 to present)

\section{Sources of literature}

Ageline (EBSCO; 1978 to present)

CINAHL Complete (EBSCO; 1937 to present)

EBM Reviews (OVID; 1991 to present)

Ergo-Abs (Ergonomics Abstracts, EBSCO; 1985 to present)

MEDLINE (Ovid; 1950 to present)

SPORTDiscus (EBSCO; 1830 to present)

Web of Science (Thomson Reuters; 1898 to present)

Clinical trial registries:

- Clinicaltrials.gov

- Controlled-trials.com

Theses/dissertations:

- ProQuest Theses and Dissertations

Abstracts/conference proceedings for target associations:

- Bioengineering

- Annual Conference of the IEEE Engineering in Medicine and Biology Society

- ASME Summer Bioengineering Conference

Biomechanics

- Annual Conference of the American Society of Biomechanics

- Biennial Meeting of the Canadian Society of Biomechanics

- Congress of the International Society of Biomechanics

Falls prevention

- Biennial Conference of the Australian and New Zealand Falls Prevention Society

- International Conference on Fall Prevention and Protection

- International Society for Posture and Gait Research World Congress

Gerontology

- Canadian Association on Gerontology Annual Scientific and Educational Meeting

- Gerontological Society of America's Annual Scientific Meeting

- World Conference of Gerontechnology

- World Congress of the International Association of Gerontology and Geriatrics

Websites of target organisations:

- Agency for Healthcare Research and Quality (AHRQ)

- American Society for Testing and Materials (ASTM) International

- Canadian Agency for Drugs and Technologies in Health (CADTH)

- Occupational Safety and Health Administration (OSHA)

- OpenSIGLE

- Parachute Canada

- The National Institute for Occupational Safety and Health (NIOSH)

- UK Health Technology Assessment

- US Center for Health Design

- WHO Health Evidence Database (HEN)

Reference lists of all eligible records

Tables of contents of 1-2 key journals

We will consult with content experts and the Research Advisory Panel to identify individual records not already uncovered by our academic database, grey literature and hand searches.

NA, not applicable. 
the approach to grey literature. At each stage of the search process, an information scientist (ACD) will review the proposed search strategies to ensure optimal use of terms, operators and search functionality. Each month the project team will receive automatic alerts from the academic databases of recently indexed records retrieved by the search strategy to ensure that the search results are current. All records identified through licensed electronic database searching will be saved to RefWorks, an online citation management software, and duplicate citations will be removed.

\section{Grey literature}

We will conduct a targeted search of grey literature to find clinical trials in progress, abstracts and conference proceedings from pertinent scientific meetings, as well as theses, dissertations and reports not indexed in licensed academic databases (table 2). The selected sources of grey literature were chosen by content experts on the research team, in consultation with knowledge users on the Research Advisory Panel. We will implement a variety of search strategies to obtain relevant records from each source, including manually screening titles, Google advanced search, Adobe Reader advanced search and website search engines. We will keep our search terms intentionally broad (eg, floor, surface, elder, senior, fall, injury) to keep this initial grey literature search as inclusive as possible.

\section{Hand searching and consultation with experts}

The references from all records that are identified for inclusion in our review will be manually reviewed. We may also hand search tables of contents from 1990 until present day of one or two key journals to identify records that may have been missed in database and reference list searches. These journals will be identified during the data charting stage as those that yield the highest numbers of eligible records. We will also consult with the Research Advisory Panel and relevant academic experts to uncover additional relevant literature.

\section{Identifying relevant records \\ Eligibility criteria}

We seek to include records that describe the biomechanical efficacy, clinical effectiveness, cost-effectiveness, and workplace safety of compliant flooring systems that aim to prevent fall-related injuries (table 1). Records will be selected according to the criteria below. ${ }^{31}$

Designs: Consistent with scoping review methodology, we will consider all methodological designs (ie, primary and secondary research), including published and unpublished records of quantitative, qualitative or mixed-methods research design. We will exclude marketing materials from flooring manufacturers, such as product guides, which may present biased information.

Participants: We will include records that include human participants 18 years of age or older (eg, university students, older adults). We will also include laboratory tests of compliant flooring, economic analyses and other records that may not involve human participants. We will exclude all records that exclusively examine animals, children/teens ( $0-17$ years) or athletes (all ages) as findings from these populations would be unlikely to influence decision-making about adoption of compliant flooring in healthcare settings for older adults.

Interventions: We will include records if they examine any of the flooring types included in our definition of 'compliant flooring systems'. This includes safety flooring, shock absorbing flooring, dual stiffness flooring, rubber flooring, acoustic flooring and carpet. Fall mats will not be considered a compliant flooring system for several reasons: they are not permanently affixed to the floor; they do not provide universal coverage or protection; and they are considered to be programmatic equipment. Thus, studies reporting exclusively on fall mats will not be eligible.

Comparators: Given the broad range of interventions of interest, several comparisons will be relevant to include. ${ }^{31}$ We anticipate that the most common comparator will be a rigid flooring condition (eg, concrete or $<2 \mathrm{~mm}$ vinyl/resilient sheeting). In addition, we will describe other effect modifiers (eg, age group, setting type, body mass index) that were measured in the primary studies.

Outcomes: Outcomes of interest will vary depending on the theme(s) of the record. We will consider (but not necessarily limit to) the following outcomes for each theme:

Biomechanical efficacy: we will consider records that report evidence from laboratory research about (1) impact force attenuation or energy absorption during real or simulated falls onto compliant flooring systems, or (2) gait performance, mobility performance, assistive device use and/or balance on compliant flooring systems.

Clinical effectiveness: we will consider records that examine how compliant flooring systems affect falls and fall-related injuries.

Cost-effectiveness: we will consider records that provide evidence related to the cost of compliant flooring systems (eg, material and installation costs, longevity of flooring) relative to their effects on fall and fall-related injury healthcare costs. We expect that most records on cost-effectiveness will report their results as costbenefit ratios and/or cost-effectiveness ratios.

Workplace safety: we will consider records that provide evidence about the effects of compliant flooring systems on musculoskeletal health and fatigue of healthcare workers as a direct result of differences in floor compliance. Records may include perceived/subjective ratings of systemic and musculoskeletal fatigue or objective measures of fatigue (eg, via physiological sampling) while working on compliant flooring. We will not include records reporting on workplace hazards/safety associated with floor traction or floor slipperiness. 
Setting: Records will be included if the research is conducted in a laboratory, community or relevant healthcare setting (eg, acute care, assisted living, LTC). Consistent with our population exclusion criteria, we will exclude records if the research was conducted within a sporting, playground, school or paediatric acute care setting.

Timing and language: We will not limit our academic database search by year or language of publication. For records not available in English, we will use Google Translate or a paid translator, when necessary. The grey literature search will be limited to English language records published after 1990, when the first records on the biomechanical efficacy of compliant flooring were published (informed by research team content experts).

\section{Selection process}

We will select relevant records using a two-step process: (1) review titles and abstracts (level 1 screening) and (2) review full-text records (level 2 screening). We will use a hierarchical approach when screening records. We will first exclude records if they are marketing materials or if they describe research exclusively involving animals. Next, we will exclude records that do not include at least one type of compliant flooring system; and then we will exclude records that do not address one of the four themes. Lastly, we will exclude records if the population of interest is exclusively children and/or athletes.

Level 1 screening of licensed academic databases (titles and abstracts): We will conduct level 1 screening in a similar way to Pitzul et al. ${ }^{32}$ First, we will perform a pilot test on a random sample of 50 records in which two independent reviewers (CCL and MPJ) will screen the titles, abstracts and descriptors based on the defined eligibility criteria using a level 1 screening form. ${ }^{32}$ An independent research assistant will compare the results from the two reviewers to identify discrepancies. The two reviewers will attempt to resolve the discrepancies and will involve a third reviewer knowledgeable in the area (DCM), if necessary. Following the pilot, if the reviewers identify types of record or portions of the screening form that are more prone to discrepancies, eligibility criteria and the level 1 screening form may be revised and further pilot tests may be performed to ensure consistent application of the eligibility criteria.

Following the pilot(s), the two independent reviewers will screen the remaining records using the level 1 screening form. Records will be screened in batches of $\sim 700$ and discrepancies will be identified by an independent research assistant and resolved by the two primary reviewers, with help from a third if necessary, after the completion of each batch. This will allow us to periodically identify and correct any systematic differences in application of the eligibility criteria between the two reviewers. In situations when there is not enough information to make an informed decision about inclusion/exclusion, the record will be retained for level 2 screening. To further increase the study's rigour, a third reviewer (DCM) will rescreen $5 \%$ of records excluded by the other two reviewers to confirm that eligibility criteria were applied appropriately.

Level 2 screening of licensed academic databases (full-text review): Full texts of all records that pass level 1 screening will be retrieved, and we will apply the same inclusion/ exclusion criteria. We will again perform a pilot test, this time on 20 records. Records will then be independently screened by both reviewers in batches of 50 . Discrepancies will be identified by an independent research assistant and resolved by the two primary reviewers, with help from a third if necessary, after the completion of each batch. Reviewers will also categorise records into the four themes at this stage.

Grey literature screening: We will also perform grey literature screening in two stages, applying the same inclusion/exclusion criteria as described for the licensed academic database screening. First, one reviewer (MPJ) will search through identified grey literature sources for relevant records (table 2). If there is not a definitive reason for exclusion, the reviewer will include the record. A second reviewer (CCL) will then screen the records for any other necessary exclusion.

\section{Charting the data}

In consultation with our Research Advisory Panel, an electronic data charting form will be designed and used by two independent reviewers (CCL and MPJ) to extract key information from full-text records that pass level 2 screening (table 3). As a pilot, the reviewers will chart data from a random sample of four records to determine whether their approach to data extraction is consistent with the research questions and purposes. ${ }^{26}$ Consistent with the iterative nature of scoping studies, the form will be revised, if necessary. Reviewers will then use the final version of the data charting form to extract data from the remaining records. Since secondary research (eg, literature reviews) may include records that are already accounted for by primary research records, many of the secondary research records will be included for descriptive purposes only (ie, they will be included in the total number of records that discuss compliant flooring, but results of specific studies contained in them will not be charted if they are also presented in a primary research record). Reviewers will meet on a regular basis, throughout the data charting process, and data entry will be compared after the completion of charting each theme to determine where there are inconsistencies; discrepancies will be resolved by consensus or a third party adjudication. ${ }^{33}$

The research team acknowledges that there is a large potential for discrepancy at this stage, and therefore several strategies will be used to improve consistency of charting. First, where possible, drop-down menus will be used in data entry spreadsheets (ie, data validation) to avoid trivial discrepancies such as punctuation differences. Second, sections of records from which large amounts of data will be collected (eg, research aims, 
Table 3 Data extraction for research question

\section{Data to be extracted}

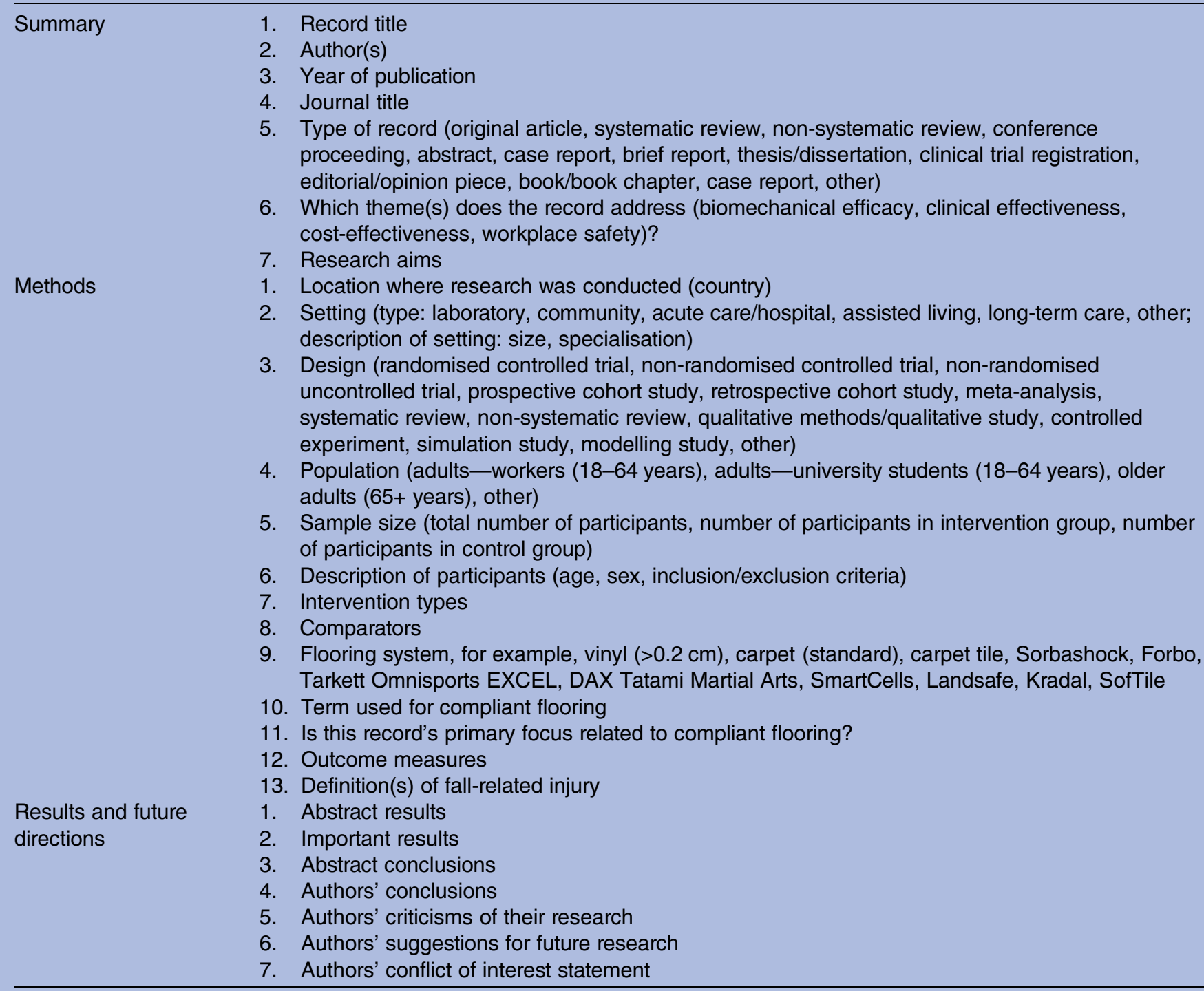

results, conclusions, etc) present a particularly high chance of inter-rater discrepancy; thus, where possible, we will transpose these data exactly as they exist in the original record. Finally, we will attempt to state results and conclusions in terms of the author's research aims. When comparing discrepancies in these sections, the reviewers will look for differences in content only and will record all relevant data from both entries into one master copy.

\section{Collating, summarising and reporting the results}

We will summarise the results of the scoping review using both numerical (quantitative) and narrative (qualitative) analysis. Our numerical analysis will map the records in terms of year of publication, country of origin, source (eg, licensed academic databases vs grey literature), methodology and key themes (ie, biomechanical efficacy, clinical effectiveness, cost-effectiveness and workplace safety). Our narrative analysis will produce a summary of the existing records related to compliant flooring systems for fall injury prevention in older adults within each of the four themes. Within each theme, we will synthesise the evidence according to questions the Research Advisory Panel deems pivotal to aid in decision-making. We will also describe strengths and limitations of the evidence available within each theme. Our synthesis of results will convey the breadth of the field, illustrate the dominant areas of research in the field, and allow us to uncover locations of significant gaps in research, which will inform a list of future directions and additional areas of discussion with the Research Advisory Panel. We will also produce a concept map to detail the terms used for compliant flooring and the types (brands) of compliant flooring being used in research. Study quality will not be assessed in this scoping review, consistent with guidance on scoping 
review conduct; ${ }^{27} 34{ }^{35}$ results from studies that lack quality may introduce bias in our study's findings. ${ }^{26}$ Together, the summarised results will be disseminated to key stakeholders through our planned knowledge translation efforts.

\section{Consulting with stakeholders}

Our review will be informed at each stage by iterative consultations (ie, small group meetings, one-on-one discussions, videoconferences, teleconferences) with a range of potential knowledge users. Specifically, we have formed a Research Advisory Panel composed of the following knowledge users: two managers of fall and injury prevention for local health authorities, two directors of care at LTC sites, a physiotherapist at a LTC site and two representatives from Lower Mainland Facilities Management, which manages all infrastructure projects for the four Lower Mainland health authorities in British Columbia. Collectively, members of the Panel possess the relevant expertise and decision-making authority to critically evaluate and implement compliant flooring systems in high-risk environments such as LTC.

An interactive process of communication between researchers and the Research Advisory Panel will be used throughout all stages of the scoping review process. We will involve the Panel in a number of important ways: (1) in providing input on the design and implementation of the review; (2) as members of the project team who attend semiregular project meetings; (3) in the interpretation of findings and identification of research gaps; and (4) in the packaging and dissemination of the review's findings in a form that is relevant, practical and easily interpreted by other decision-makers and knowledge users.

\section{ETHICS AND DISSEMINATION}

Our end-of-grant dissemination plan matches knowledge translation strategies to each of our target audiences to support the advancement of health-related knowledge. To disseminate findings to stakeholders from a range of sectors, and to capitalise on the power of in-person communication, we will host a 1-day Stakeholder Symposium to which we will invite individuals representing healthcare, occupational health, building design and construction, government, housing, flooring manufacturers, and research. To disseminate findings to a broader geographic audience of stakeholders from a range of sectors, we will host a webinar and produce and distribute a Knowledge-to-Action Report. To reach academic stakeholders, we will prepare and submit both a conference abstract and open-access peer-reviewed journal article with the study results. We will evaluate the success of our end-of-grant dissemination efforts through a variety of means, such as tracking event attendance, event evaluation forms, downloads and citations of our scientific journal article, and distribution numbers for the Knowledge-to-Action Report. The review's findings and outputs will be a first step to help decision-makers understand the current evidence base on compliant flooring that aims to prevent fall-related injuries.

In conclusion, this scoping review responds to the information needs of healthcare decision-makers tasked with preventing fall-related injuries by synthesising the available evidence about compliant flooring as a potential intervention for preventing fall-related injuries in older adults as well as by identifying gaps in evidence and new avenues for research. Results will be especially useful in LTC, but also applicable in acute care, assisted living and home care.

Twitter Follow Chantelle Lachance at @_CLachance

Acknowledgements The authors would like to express gratitude to the Research Advisory Panel for their invaluable consultations and engagement with the research project thus far. The Panel includes the following content experts and knowledge users: Dr Stephen Robinovitch (Professor, Simon Fraser University), Dr Fabio Feldman (Manager-Older Adults Program, Fraser Health Authority), Tanya Dunne (Regional Program Lead-Fall and Injury Prevention Program, Vancouver Coastal Health Authority), Shannon Johnson (Director of Care, Revera), Leslie Karmazinuk (Chief Operating Officer, New Vista Society Care Home), Pet Ming Leung (Physiotherapist), Maureen Haddock (Facilities Planner, Lower Mainland Facilities Management) and Rob Kolen (Project Manager, Lower Mainland Facilities Management).

Contributors CCL was involved in project conception, preliminary literature review, development of search strategy, writing and editing the protocol, and content expert input (compliant flooring). MPJ was involved in writing and editing the protocol and preliminary literature review. ACD was involved in the development of the search strategy and editing the protocol. DCM is the guarantor and was involved in project conception, preliminary literature review, development of search strategy, writing and editing the protocol. All authors read, provided feedback and approved the final manuscript for submission to BMJ Open. ${ }^{31}$

Funding This study was supported by a Knowledge Synthesis Grant from the Canadian Institutes of Health Research (KRS-140996 to DCM). CCL is a recipient of Canadian Institutes of Health Research (CIHR) Frederick Banting and Charles Best Canada Graduate Scholarships and AGE-WELL Graduate Student Award in Technology and Aging. MPJ received support from an Undergraduate Research Prize from the Department of Biomedical Physiology and Kinesiology at Simon Fraser University. DCM received support from a Michael Smith Foundation for Health Research Scholar Award.

Competing interests None declared.

Provenance and peer review Not commissioned; externally peer reviewed.

Open Access This is an Open Access article distributed in accordance with the Creative Commons Attribution Non Commercial (CC BY-NC 4.0) license, which permits others to distribute, remix, adapt, build upon this work noncommercially, and license their derivative works on different terms, provided the original work is properly cited and the use is non-commercial. See: http:// creativecommons.org/licenses/by-nc/4.0/

\section{REFERENCES}

1. Centers for Disease Control and Prevention, National Center for Injury Prevention and Control. Web-based Injury Statistics Query and Reporting System (WISQARS). Atlanta, GA, 2015. http://www. cdc.gov/injury/wisqars (accessed 28 Feb 2016).

2. Parachute. The cost of injury in Canada. Toronto, ON, 2015:26 http://parachutecanada.org/downloads/research/Cost_of_Injury-2015. pdf (accessed 28 Feb 2016).

3. Stevens JA, Corso PS, Finkelstein EA, et al. The costs of fatal and non-fatal falls among older adults. Inj Prev 2006;12:290-5.

4. Public Health Agency of Canada. Seniors' falls in Canada: second report. Ottawa, ON, 2014. http://www.phac-aspc.gc.ca/seniors-aines/ 
publications/public/injury-blessure/seniors_falls-chutes_aines/assets/ pdf/seniors_falls-chutes_aines-eng.pdf (accessed 28 Feb 2016).

5. Rubenstein LZ, Josephson KR, Robbins AS. Falls in the nursing home. Ann Intern Med 1994;121:442-51.

6. Tinetti ME, Speechley M, Ginter SF. Risk factors for falls among elderly persons living in the community. N Engl J Med 1988;319:1701-7.

7. O'Loughlin JL, Robitaille $\mathrm{Y}$, Boivin JF, et al. Incidence of and risk factors for falls and injurious falls among the community-dwelling elderly. Am J Epidemiol 1993;137:342-54.

8. Stevens JA, Olson S. Reducing falls and resulting hip fractures among older women. Home Care Provid 2000;5:134-9; quiz 140-1.

9. Tinetti ME, Speechley M. Prevention of falls among the elderly. N Engl J Med 1989;320:1055-9.

10. Butler M, Norton R, Lee-Joe T, et al. The risks of hip fracture in older people from private homes and institutions. Age Ageing 1996;25:381-5.

11. Wolinsky FD, Fitzgerald JF, Stump TE. The effect of hip fracture on mortality, hospitalization, and functional status: a prospective study. Am J Public Health 1997:87:398-403.

12. Norton R, Butler M, Robinson E, et al. Declines in physical functioning attributable to hip fracture among older people: a follow-up study of case-control participants. Disabil Rehabil 2000;22:345-51.

13. Harvey LA, Close JC. Traumatic brain injury in older adults: characteristics, causes and consequences. Injury 2012;43: $1821-6$.

14. Korhonen N, Niemi S, Parkkari J, et al. Incidence of fall-related traumatic brain injuries among older Finnish adults between 1970 and 2011. JAMA 2013;309:1891-2.

15. Watson WL, Mitchell R. Conflicting trends in fall-related injury hospitalisations among older people: variations by injury type. Osteoporos Int 2011;22:2623-31.

16. Wright $A D$, Laing $A C$. The influence of headform orientation and flooring systems on impact dynamics during simulated fall-related head impacts. Med Eng Phys 2012;34:1071-8.

17. Curtis Woodford CY. Toronto notes 2013: comprehensive medical reference and review. Toronto: Toronto Notes Incorporated for Medical Students Incorporated, 2013.

18. Anderson R, McLean J. Biomechanics of closed head injury, head injury. Pathophysiology and management. London: Hodder Arnold 2005.

19. Cameron ID, Gillespie LD, Robertson MC, et al. Interventions for preventing falls in older people in care facilities and hospitals. Cochrane Database Syst Rev 2012;12:CD005465.

20. Vlaeyen E, Coussement J, Leysens G, et al. Characteristics and effectiveness of fall prevention programs in nursing homes: a systematic review and meta-analysis of randomized controlled trials. $J$ Am Geriatr Soc 2015;63:211-21.
21. Vu MQ, Weintraub N, Rubenstein LZ. Falls in the nursing home: are they preventable? J Am Med Dir Assoc 2006;7:S53-8.

22. Kannus P, Parkkari J, Niemi S, et al. Prevention of hip fracture in elderly people with use of a hip protector. $N$ Engl $J$ Med 2000;343:1506-13.

23. Cameron ID, Cumming RG, Kurrle SE, et al. A randomised trial of hip protector use by frail older women living in their own homes. Inj Prev 2003;9:138-41.

24. O'Halloran PD, Cran GW, Beringer TR, et al. A cluster randomised controlled trial to evaluate a policy of making hip protectors available to residents of nursing homes. Age Ageing 2004;33:582-8.

25. Bentzen $\mathrm{H}$, Forsén $\mathrm{L}$, Becker $\mathrm{C}$, et al. Uptake and adherence with soft-and hard-shelled hip protectors in Norwegian nursing homes: a cluster randomised trial. Osteoporosis Int 2008;19:101-11.

26. Levac D, Colquhoun H, O'Brien KK. Scoping studies: advancing the methodology. Implement Sci 2010;5:1-9.

27. Peters MD, Godfrey CM, Khalil H, et al. Guidance for conducting systematic scoping reviews. Int J Evid Based Healthc 2015;13:141-6.

28. Arksey H, O'Malley L. Scoping studies: towards a methodological framework. Int J Soc Res Methodol 2005;8:19-32.

29. Daudt HM, van Mossel C, Scott SJ. Enhancing the scoping study methodology: a large, inter-professional team's experience with Arksey and O'Malley's framework. BMC Med Res Methodol 2013;13:48

30. Colquhoun HL, Levac D, O'Brien KK, et al. Scoping reviews: time for clarity in definition, methods, and reporting. J Clin Epidemiol 2014;67:1291-4.

31. Shamseer L, Moher D, Clarke M, et al. Preferred reporting items for systematic review and meta-analysis protocols (PRISMA-P) 2015: elaboration and explanation. BMJ 2015;349:g7647. doi:10.1136/bmj. g7647 [doi].

32. Pitzul KB, Munce SE, Perrier L, et al. Quality indicators for hip fracture patients: a scoping review protocol. BMJ Open 2014;4: e006543.

33. McArthur C, Gibbs J, Papaioannou A, et al. Scoping review of physical rehabilitation interventions in long-term care: protocol for tools, models of delivery, outcomes and quality indicators. BMJ Open 2015;5:e007528. doi:10.1136/bmjopen-2014-007528 [doi]

34. Pham MT, Rajić A, Greig JD, et al. A scoping review of scoping reviews: advancing the approach and enhancing the consistency. Res Synth Methods 2014:5:371-85.

35. Tricco AC, Lillie E, Zarin W, et al. A scoping review on the conduct and reporting of scoping reviews. BMC Med Res Methodol 2016;16:15.

36. Lachance CC, Feldman F, Laing AC, et al. Study protocol for the Flooring for Injury Prevention (FLIP) Study: a randomised controlled trial in long-term care. Inj Prev 2016. doi:10.1136/injuryprev-2016042008 